\title{
Design and implementation of security efficient reliable routing protocol for wireless sensor network
}

\author{
Weixia Guo \\ Department of Information Engineer \\ Guangdong Polytechnic \\ Foshan, China \\ e-mail: guoweixiaedu@163.com
}

\begin{abstract}
The generic protocol stack for wireless sensor networks including: physical layer, data link layer, network layer, transport layer, application layer corresponding to the five layer protocol of the internet. Design of wireless sensor network routing protocol is affected by the characteristics of network, data fusion technology and the influence of many factors such as node localization technology. This paper firstly compares and analyses the traditional routing protocol for wireless sensor networks. This paper presents design and implementation of security efficient reliable routing protocol for wireless sensor network. Experiments show that the proposed routing protocol is effective.
\end{abstract}

Keywords- Wireless sensor network; Routing protocol; Sensor node; Low-energy adaptive clustering hierarchy; Simulation

\section{INTRODUCTION}

Wireless sensor network (WSN) is a kind of wireless ad hoc network special, it is a kind of network application system composed of a large number of densely deployed sensor nodes form in intelligent monitoring area. The fast and convenient deployment characteristics and complete monitoring ability which is widely used in military, industrial process control. Stack protocol of wireless sensor network mainly consists of physical layer, data link layer, network layer, transport layer and application layer, the five layers of the internet protocol and corresponding.

Wireless sensor network is especially suitable for deployment to arrive in harsh environment or human should not be area, nodes need to work in the open air environment, it requires nodes stronger, able to adapt to a variety of harsh environments [1]. Secure communication and network security is very important; in order to prevent the monitoring data from being stolen and forged insert monitoring information. Therefore, the software and hardware of the wireless sensor network must have the robustness and fault tolerance.

The collaborative nature of wireless sensor network, explains the importance and necessity of self organization mechanism. In the self organization, and it is node local information and such as energy or processing capacity of these characteristic into consideration to decide and its neighbor node's local communication structure. All of the node's local decision will cause a new behavior: organization wide network. In wireless sensor networks, because of limited energy of sensor nodes, energy or energy efficiency is an important issue to consider.

The traditional routing protocols affect the network life cycle. Energy in wireless sensor networks based on limited, saving energy is one of the main problems in network design, an important goal of topology control is to guarantee network connectivity and coverage of the case, to reasonably and efficiently use the network energy, and prolong the network survival time.

High density deployment of sensor nodes, network topology changes quickly, for the maintenance of topological structure and also challenge. These features make the wireless sensor network is a self-organizing network different from traditional, and obtained the outstanding performance in some architecture design attempts in current. This paper presents design and implementation of security efficient reliable routing protocol for wireless sensor network.

\section{ANALYSIS AND COMPARISON OF THE TRADITIONAL} ROUTING PROTOCOL FOR WIRELESS SENSOR NETWORKS

Compared with the traditional routing technology in Ad hoc network, routing algorithm for wireless sensor networks require special consideration in the design of the energy consumption problem. Energy saving based routing has several kinds, such as the maximum available power (PA) routing algorithm, namely routing the total effective power maximum, total effective power can be obtained by the effective power accumulation on the route [2]. Minimum energy routing algorithm, the algorithm selects the minimum energy consumption from sensor nodes to the receiver to transmit the data routing.

Monitoring information to be transmitted to the management server, a user-friendly real-time query; 4 , the gateway of wireless communication: because of the particularity of the monitoring region, there is not necessarily a wired network, for example: Internet, wired local area network, the gateway has put environmental information wireless acquisition transmission to the server function is not affected, the monitoring region accuracy: sensor determines the data acquisition accuracy, sensor 
data acquisition accuracy can be within the specified error range of memory in a certain degree of it.

The flood routing (Flooding): This is a kind of traditional network routing protocol. The algorithm does not require the maintenance of computing the topology of the network and related routing, nodes receive information is required only to broadcast forwarding packets. For example: the $\mathrm{S}$ node to transmit a data to the $\mathrm{D}$ node, it needs through the network transfers copies to each of its neighbor nodes, until transferred to node $\mathrm{D}$ far or for the data set to zero until the survival period, as is shown by equation (1) [3].

$$
\overline{C E N}=\sqrt{\left(C E N_{1}^{2}+C E N_{2}^{2}\right) / 2}
$$

SPIN (Sensor Protocol for Information via Negotiation) protocol is a data centric adaptive routing protocol. The SPIN protocol is designed to: nodes through consultation between the Flooding protocol and Gossiping protocol to solve the implosion and overlap. SPIN protocol has 3 types of message, namely ADC, REQ and DATA, ADC for data broadcast, when a node has data can be shared, can carry on data information broadcast by the. REQ is used to request to send data, when a node wants to accept DATA packets, sending REQ packets. DATA as the sensor collected data packet.

According to the route selection is considering the QoS constraints, can be divided into the guarantee of QoS routing protocol and QoS routing protocol does not guarantee. Ensure that the QoS routing protocol is refers to the route is established, considering the delay and packet loss rate of QoS parameters, choose a most suitable for QoS application request routing from many feasible route.

Flooding algorithm and gossiping algorithm is the two classic in wireless sensor network algorithm, compared with other routing algorithms, they are less dependent on the network topology. The flooding algorithm, each node receives data packets will send this packet broadcast to all its neighbor nodes, until the packet reaches the destination node or reach the maximum number of hops. Gossiping algorithm by improving on the flooding algorithm, the received data packet node random choice is not a data sending neighbor node, the data packet transmission in the past, through the continued implementation of the process to achieve the purpose of communication data.

SMACS (Self-Organizing Medium Access Control for Sensor Networks) protocol is a distributed protocol, without any global or local master node, can be found in neighboring nodes and the establishment of transmit / receive dispatch table. The slot chain routing selection of random and fixed frequency is composition. Although each subnet neighbor node communication need time synchronization, but does not need to synchronize the whole network. The use of a random wake-up mechanism in the linking stage, turn off the wireless transceiver device in my spare time, to achieve the purpose of energy saving.

$$
\tilde{x}(j \mid k)=x(j)-\hat{x}(j \mid k)
$$

This agreement is required to complete the following basic functions: a) hops in the region of the sensor node network joint point; b) at a predetermined time $\mathrm{T}$, the isolated state of sensor nodes (nodes that did not join the $S$ routing backbone) may receive the Adv message, Adv message node distance parameter all received in the $S$ view the distance parameter, node will be the smallest (1) as the parent node itself, at the same time distance parameters of parent node is saved to the parent node in the collection and record yourself to the gateway node value (i.e. distance parameters of the parent node value plus 1 ), then the distance parameter Adv message in the increase of 1, continued to broadcast the Adv message at the same time, if node a has joined the routing backbone Adv message is received, it is the parent node set update.

In wireless sensor networks, should offer application layer protocol and how to provide the key is still a problem. The application layer protocol includes a sensor network management protocol (Sensor Networks Management Protocol, SNMP), delegate tasks and data Advertising Protocol (Task Assignment Data Advertisement Protoco1, TADAP) and sensor query and data dissemination protocol (Sensor Query and Data Dissemination Protocol, SQDDP), different from the traditional protocol, sensor network management protocol SNMP required to solve the sensor node has no global identification number problem, support attribute names or named mode based on [4]. The main function of sensor network management protocol of SNMP include: configuration management, fault management, performance monitoring and security management.

The SPIN algorithm does not explicitly define metadata format. The specific format and application, such as ADV and REQ format according to the specific application to do the detailed provisions. In addition, when the topology changes, each node only needs to maintain the state of its neighboring nodes in the local scope, without the whole network broadcast, both to save energy and reduce the node processing capacity requirements, this kind of SPIN is called SPIN-1, as is shown by equation (3).

$$
f_{u}^{(2)}=u^{T} H u
$$

SPA algorithm selects a set of nodes in the network with the maximum density as establishing network coordinate system of the reference point, and in which the choice is communicated with a maximum node degree as the origin of the coordinate system. Firstly, according to the results of ranging between nodes in each node to establish local coordinate system, through the exchange of information between nodes and coordination, to the point of reference for reference through coordinate transformation and gradually establish the global coordinate system. The main drawback of the SPA algorithm is the network communication overhead is too large.

Directed Diffusion model of Estrin et al. Specifically for the routing strategy is designed for sensor networks, node group with a < attribute, value $>$ to name it generated data. The Sink node queries issued business interests (interest) to say, interest is a group < attribute, value > combination, the gradual spread, eventually traversal of the whole network, find all the matching the original data. Known as a "gradient" variables and the whole business 
request diffusion process connected, reflects the approximate judgement intermediate network node matching request condition data source.

\section{APPLICATION OF WIRELESS SENSOR NETWORK ROUTING PROTOCOL}

In wireless sensor networks usually have one or more node acts as a data sink (Sink), the sensor nodes collect data in the network, through multi hop and transmitted to Sink, Sink will be the fusion data transmitted through wired or wireless mode to the observer.

Wireless sensor networks are usually placed in no base structure, node needs to have the ability of selforganization, can automatically configure and manage, and through the topology control mechanism and network protocols to form a multi hop wireless network data forwarding. Application of correlation network, wireless sensor network is used for sensing the objective physical world, physical world and information acquisition. Physical quantity of the objective world is varied, not exhaustive.

The emergence of wireless sensor networks to provide convenience for the study of data random access, and can also avoid intrusive to the environment from the destruction of traditional data collection [5]. Wireless sensor networks are also used in some other areas. For example, some dangerous industrial environments such as mine, nuclear power plant, and the staff can use it to implement safety monitoring. In addition, and also in many fields such as industrial automation production line, as is shown by equation (4).

$$
G(x, y, \sigma)=\left(2 \pi \sigma^{2}\right)^{-\frac{1}{2}} \exp \left(-\frac{x^{2}+y^{2}}{2 \sigma^{2}}\right)
$$

In the SPIN-2 joined the energy threshold mechanism based on SPIN-1. In the delivery of first detection of adjacent nodes energy data, if the energy value is lower than a certain threshold value, the node is considered to not have enough ability to complete the remote delivery task, the data is forwarded to other neighboring nodes enough energy. In addition, according to the different application environment, other extended protocol of SPIN, such as SPIN- BC and SPIN-RL in the sensor network the multicast network, SPIN-PP and SPIN-EC according to the traditional point to point network make special optimization.

Multi hop routing, nodes by the communication distance, power control or energy limitation, when a node cannot direct communication with the gateway, required by other nodes forwarding the data transmission, and so the network data transmission is multi hop routing. Automatic monitoring system is for agricultural environment. Various environmental factors including water, electricity, heat, light, for agricultural production is very important, effective monitoring of these environmental factors based on the wireless sensor networks, and acquisition control appropriate environment related data, can improve the degree of intensive agriculture production, simplify the system complexity, reduce the cost of the device, effectively improve the efficiency and effectiveness of agricultural production.
In some easy occurrence of debris flow, landslides and other natural disasters place, use monitoring wireless sensor network in a timely manner, long-term terrain changes, to those places of various environmental factors, and the acquisition of the appropriate analysis of relevant data, when the disaster will occur, we can advance warning reports to prepare or take corresponding measures to prevent their further occurrence.

Sensor network has some successful examples in medical field. SSIM (Smart Sensors and Integrated Microsystems) project, 100 micro sensors is placed in the patient's eyes, so as to help the blind get a certain degree of vision [6]. With the help of various medical sensor networks, people can enjoy more convenient and comfortable medical service.

$$
\operatorname{pr}\left\{m_{i}(k+1) \mid m_{j}(k)\right\}=\pi_{j i}
$$

Architectural and urban management, wireless sensor network for monitoring the health status of the building, not only low cost, but also can solve the traditional monitoring complex wiring, circuit ageing, vulnerable to damage and other issues.

All other nodes by selecting appropriate from the auxiliary nodes to coordinate calculation of the local coordinate system of node in the domain. The relevant coordinate information domain and then adjacent nodes rely on joint marginal node two domains, with the main node ID smaller local coordinate system as the reference, the coordinate's conversion between adjacent local coordinate system, to the establishment of a global coordinate system. Compared with SDGPSN algorithm and SPA algorithm, the communication overhead is significantly reduced, which makes it more suitable for large-scale deployment in wireless sensor networks.

In the converging point send enhanced information to establish the main path $\mathrm{P}$, suboptimal path to enhance convergence point send message to the secondary node A, node A to select the optimal node B to suboptimal path to enhance message passing down [7]. If the $B$ in the main path of $\mathrm{P}$, then $\mathrm{B}$ send a negative message to the A A enhancement, in the transmission of suboptimal path to enhance information to suboptimal node another; if the B is not on the main path continues to transfer suboptimal path to enhance information, as is shown by equation(6).

$$
\hat{r}=\sum_{i=N_{1}}^{N_{2}} d^{*}(i) x^{H}(i)
$$

Directed Diffusion uses a demand based data query mechanism. That is to say, in Directed Diffusion, by Sink node sends data query request, whereas in SPIN, the node broadcasts its own data, to allow other nodes to query. Directed Diffusion has many advantages. For example, nodes only need to communication and node address mechanism and therefore does not require a global; each node can perform data fusion operation, in order to save the energy consumption; node does not need to maintain the topology of the network, data transmission is based on the demand, so it is a very energy-efficient routing protocol. 
Although the sensor network is based on the physical layer of relatively stable, but its main work is still collecting, processing and statistical data. The design is based on the data management and processing center, consisting mainly of network data storage, query, analysis, mining, and according to the initial understanding of data processing decision making. Therefore, wireless sensor network is a data centric network. At the same time, wireless sensor network routing protocol is to deal with network data as the main objective.

\section{DESIGN AND IMPLEMENTATION OF SECURITY EFFICIENT RELIABLE ROUTING PROTOCOL FOR WIRELESS SENSOR NETWORK}

This paper proposes an energy efficient security and trusted routing protocols in wireless sensor network, and it is in the LEACH simulation environment PEGASIS network delay and the average time of this Agreement and the average energy consumption was compared and analyzed the performance parameters.

In the hierarchical structure of the network, the network node has some association composed of clusters (Cluster). In the clusters, usually with an elected according to certain rules and it is known as the cluster head (Sink) node. In addition to the Sink node, the general node member functions are relatively simple, do not need to maintain routing information complex. This greatly reduces the routing control information, and has good extensibility; its drawback is that Sink may become the bottleneck of the network.

At present, NS2 has been used to simulate all kinds of network researchers widely. NS-2 is an object-oriented, discrete event driven network simulator, is mainly used to solve the problem of research of the network. NS-2 offers a wireless or wired network, simulation of TCP, routing, multicast protocols.

Trust factor communication aspects are the main factor to consider trust evaluation system. In wireless sensor networks, transmission of control commands and sensing data is the main node behavior can be observed. A malicious node may selectively dropping packets forwarding, tampering with the message, etc.. Selfish nodes may also be because of reducing energy consumption and reduce network participation, need to forward the packet, reducing the number of packets sent as discarded.

Protocol self organization has higher energy efficiency in the initial stage; in the maintenance of energy efficiency may not be very high stage [8]. Compared with other protocols, although in the initial stage, can form these organizational structure with less energy, but they may need more information interaction, consume more energy, in order to maintain the perplexing structure. On the contrary, the organizational structure of a concise and takes more energy to be set up in the initial stage, however, requires less energy to maintain.

LEACH (low-energy adaptive clustering hierarchy) is a proposed earlier WSN hierarchical routing algorithm based on clustering. With the traditional fixed network gateway node energy more adequate than WSN, the node energy is limited, therefore cannot use the same set of cluster head node as a gateway. LEACH WSN cluster head randomly selected from a few nodes, considering the balance of all the energy consumption of nodes in the network, let the other do not cluster head nodes take turns as the cluster head, so that the network is not because of a few nodes to deplete energy caused by the network paralysis.

Distributed energy aware node activity (distributed energy-aware node activation DEANA) protocol time is frame into scheduling access phase of periodic and random access stage. Scheduling access stage is composed of a plurality of continuous data transmission time slot, a slot is allocated to a particular node to send data. In addition to the corresponding receiving node, other nodes in this time slot in a sleep state [9]. Random access stage switch is composed of a plurality of continuous signaling time slot, used in the treatment of node adding, deleting and time synchronization as is shown by equation (7).

$$
F S=a b s\left(\frac{M I_{F A}}{M I_{F A}+M I_{F B}}-0.5\right)
$$

The algorithm is based on the DV-Hop locating algorithm, using a mobile beacon node with a GPS positioning device in the network according to a predetermined mobile path and continue to broadcast their location information, forming a plurality of virtual beacon, the unknown node records to the hop count every virtual beacon, the average hop distance by weighted processing to re calculate the applicable to the regional network average hop distance and the mobile beacon broadcasting, again with the hop number calculated by multiplying the distance with each virtual beacon, and finally calculate the position information by using the improved three edge measurement method, to achieve precise positioning of node.

Hierarchical PEGASIS protocol is an extension to PEGASIS, the protocol's goal is to reduce the data packet to the delay caused by the convergence point in transmission. To this end, agreement adopted data parallel transmission mechanism, and puts forward two methods to avoid conflict between sensors and signal interference may exist.

TinyOS Beaconing protocol, this protocol is simple, first of all the nodes are addressing, sink point flooding periodic routing update message, node signal coverage within the scope of the update message received, the node sends messages as the parent node is saved to the routing table, and the message is broadcast in the physical channel, so as to constitute a sink point to as the breadth first spanning tree root [10]. In this way the small scale network is simple, easy to use, but in the larger network will lead to node and sink points to jump between the increases in the number of it.

In the LEACH algorithm, the process of processing data is cluster aggregation and data fusion, in order to keep the energy of node in cluster equilibrium consumption, the selection of cluster head using the method of random distribution. In this way, each node to become a cluster head probability is 0 or 1 , if the node has some value is lower than the threshold, it will become the scolex, unlike the LEACH protocol, the PEGASIS tectonic link to execute multiple paths, data communication with the base station node selection only link. 
The system using NS-2 simulation environment, the initial state, were randomly placed into 1500 sensors in the $500 \mathrm{~m} * 500 \mathrm{~m}$ space, each sensor with $100 \mathrm{~m}$ fixed wireless coverage, the initial state of the battery energy of $8 \mathrm{~J}$. In order to test the performance of the agreement, the transmission rate in the experiment data source configuration in 640bps 2048bps, sensor bandwidth is set to $87.2 \mathrm{kbps}$. In order to test the capabilities of this agreement rerouting, the experiment will regularly add new sensors in the region during the test.

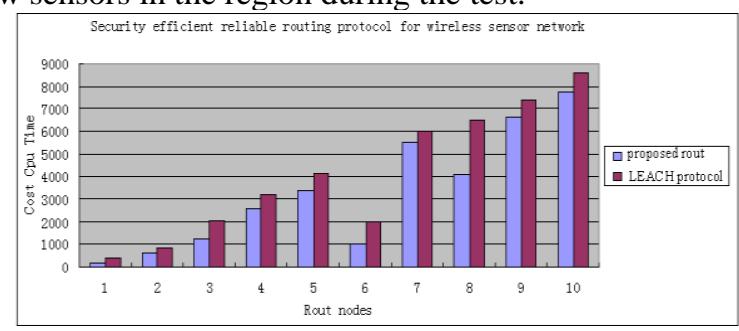

Figure 1. Comparison implementation of security efficient reliable routing protocol for wireless sensor network.

As you can see from Fig .1 only efficient node left in the 600 round, which is advanced the initial node energy is relatively high. This is because the probability of every new proposed rout protocol in the node to become a cluster head is exactly the same as that of ordinary nodes, and the initial energy less so the likelihood of death is faster. Thus the last surviving nodes are basically some advanced node.

We can see that the LEACH agreement the first node death than the node of the new protocol is a new protocol is fast, stable period longer. This is because the new protocol adopts cluster head weighted selection methods, advanced node initial energy high chances of becoming a cluster head higher, thus the time of death will be more long.

\section{SUMMARY}

This paper mainly discusses the analysis and evaluation of routing protocol in wireless sensor network, and put forward and implements a new security protocol for wireless sensor networks, reliable design, and Simulation of LEACH protocol using NS2 simulation software. By using the simulation after the file, write awk code. Firstly, the end-to-end delay is analyzed, because the LEACH protocol used in broadcasting mode, no routing search, so the delay is large, the experiment results show that the proposed protocol is safe and efficient and credible with reliable performance.

\section{REFERENCES}

[1] Xu Bao, "Energy Efficient Cluster-Based Routing Protocol for Wireless Sensor Network", JCIT, Vol. 7, No. 22, pp. $101 \sim 109$, 2012

[2] Sardjoeni Moedjiono, Aries Kusdaryono, Teddy Mantoro, "Wireless Sensor Network Energy Efficient Layer based on Hierarchical Routing Protocol (LHRP) Optimization", JCIT, Vol. 9, No. 4, pp. $12 \sim 22,2014$.

[3] Bin Wang, Xiao Wang, Bo Su, "An Improved Centralized EnergyEfficient Routing Protocol for Wireless Sensor Networks", JCIT, Vol. 7, No. 23, pp. $83 \sim 91,2012$.

[4] K.Ramanan, E.BabuRaj, "Energy Conserved Routing Algorithm In Wireless Sensor Networks", JDCTA, Vol. 8, No. 6, pp. $01 \sim 12$, 2014.

[5] corresponding author Qi Yang, Yuxiang Zhuang, Hui Li, "An Multi-hop Cluster Based Routing Protocol for Wireless Sensor Networks", JCIT, Vol. 6, No. 3, pp. $318 \sim$ 325, 2011.

[6] SU Bing, HUANG Juan, "Research on a New Energy Balancing Routing Algorithm for Wireless Sensor Networks", JCIS, Vol. 3 , No. 2, pp. $42 \sim 48,2013$.

[7] Lin yong, "Research of the Communication Distance-aware Multipath AODV Routing Protocol for Wireless Sensor Networks", AISS, Vol. 4, No. 1, pp. $139 \sim$ 146, 2012.

[8] Yusheng JIANG, Fang GUAN, Chao MA, Hui WANG, "Distributed and Steady Unequal Clustering Routing Protocol for Heterogeneous Wireless Sensor Networks", JDCTA, Vol. 7, No. 9 , pp. $352 \sim 360,2013$.

[9] AL-Gabri Malek, Chunlin Li, Naji Hasan.A.H, Zhiyong Yang, Xiaoqing Zhang, "Energy Efficiency Improvement on LEACH protocol of wireless sensor networks", IJACT, Vol. 5, No. 10, pp. 1 $\sim 9,2013$

[10] Sung-Gi Choi, Erdenekhuu Norinpel, Jinho Yoo, "Energy-Efficient Cluster Based Routing Protocol in Mobile Wireless Sensor Networks for Tracking Livestock Movements in Nomadic Herding", IJACT, Vol. 5, No. 12, pp. $150 \sim 158,2013$ 